\title{
Primary angiosarcoma of chest wall inside out: A rare presentation
}

\author{
Sabha Mushtaq', Arti Sakral', Devraj Dogra', Subhash Bhardwaj', Arshad Bhat ${ }^{3}$ \\ ${ }^{1}$ Department of Dermatology, Venereology \& Leprology, SMGS Hospital, Government Medical College, University of \\ Jammu, Jammu-180001 Jammu \& Kashmir, India, ${ }^{2}$ Department of Pathology, SMGS Hospital, Government Medical \\ College, University of Jammu, Jammu-180001 Jammu \& Kashmir, India, ${ }^{3}$ Department of Radio-Diagnosis, SMGS Hospital, \\ Government Medical College, University of Jammu, Jammu-180001 Jammu \& Kashmir, India
}

Corresponding author: Dr. Sabha Mushtaq, E-mail: smqazi.gmc@gmail.com

\begin{abstract}
Angiosarcomas are aggressive, malignant tumors of vascular or lymphatic origin with high potential to local and distant spread. Cutaneous invasion of internal tumors is rarely encountered in clinical practice. We report on a patient with vascular skin lesions over anterior chest which turned out to be incursion from chest wall angiosarcoma. Alongside the case report, literature on therapeutic arsenal of angiosarcoma is reviewed. Angiosarcoma of chest wall invading into the skin has never been reported in dermatological literature. This case is intriguing as the patient had symptomless progression of the malignancy despite aggressive invasion to overlying skin and metastasis to lungs and pleura. This impresses upon the crucial role of dermatologists in suspecting cutaneous lesions which may appear skin deep but are otherwise a silent sign of grave internal disease so that search of underlying condition is sought as soon as possible to achieve better patient outcome. So the dictum is "earlier the diagnosis, better the prognosis".
\end{abstract}

Key words: Primary angiosarcoma; Cutaneous; Chest wall; Invasion; Pulmonary

\section{INTRODUCTION}

Angiosarcomas are a subtype of soft-tissue sarcomas and are aggressive, malignant endothelial tumors of vascular or lymphatic origin [1]. The clinical presentation of angiosarcoma varies depending upon the anatomic site involved. Angiosarcomas may occur in any region of the body but are more frequent in skin and soft tissue. They can also originate in the liver, breast, spleen, bone, or heart [2]. Angiosarcomas have high propensity to metastasize or infiltrate to other sites with substantial mortality rate [3]. Herein, we present the case of a 50 year old man with primary angiosarcoma of chest wall (PACW) infiltrating and colonising the skin. The interesting nature and paucity of documentation of this rare presentation in the world literature encouraged us to report the case.

\section{CASE REPORT}

A 50 year old man presented with 3 months history of painless vascular lesions over left anterior chest wall. There was preceding history of a skin coloured deep seated nodular swelling in the mammary area which over a period of 1 month developed overlying erythematous exuberant growth. This was followed by the appearance of similar lesion near the vicinity along with small satellite lesions. Patient gave history of ulceration and bleeding from both the larger lesions with no history of any penetrating injury, breast malignancy or irradiation in the past. Rest of the medical history was not significant.

On examination, an indurated, erythemato-voilaceous fungating growth with ulceration was present over the left anterior chest wall deforming the nipple. Similar

\footnotetext{
How to cite this article: Mushtaq S, Sakral A, Dogra D, Bhardwaj S, Bhat A. Primary angiosarcoma of chest wall inside out: A rare presentation. Our Dermatol Online. 2017;8(3):306-310.

Submission: 04.10.2016; Acceptance: 20.11.2016

DOI: 10.7241 /ourd.20173.88
} 
smaller sized lesion was present over supero-medial aspect of the main lesion. The surrounding normal skin showed multiple small discrete as well as coalascing papular lesions (Fig. 1). The lesion was non-tender, bled on gentle manipulation and was fixed to underlying structures. Regional lymph nodes were not enlarged.

General physical and systemic examination was unremarkable. All routine investigations were within normal limits. For histological characterisation, an incisional biopsy was obtained with differential diagnosis of cutaneous angiosarcoma, Kaposi's sarcoma, dermatofibrosarcoma protuberans and cutaneous lymphoma. Histopathology demonstrated highly vascular lesion in the dermis composed of many variable sized vascular spaces containing papillary configurations having RBCs and lined by pleomorphic cells with vesicular nuclei and prominent nucleoli. Abnormal mitotic figures were also seen (Fig. 2). The histological features were consistent with malignant vascular tumor suggestive of angiosarcoma. Immunohistochemistry (IHC) could not be done due to limitation of resources. Chest X-ray showed an area of haziness in left lower lung zone (Fig. 3), which on lateral view appeared to be extrapulmonic chest wall mass. Ultrasound of the abdomen \& pelvis was significant for pleural effusion. Contrast enhanced computerized tomography (CECT) of the chest revealed a heterogeneous enhancing lesion measuring $5.1 \times 5 \times 2.5 \mathrm{~cm}$ with non-enhancing central area of necrosis in left anterior chest wall with muscular invasion. The lesion was also seen to infiltrate the overlying skin. An ill defined enhancing nodule was seen medial to the main lesion in parasternal area. Bilateral pleural based soft tissue densities along with secondaries in both lung fields were also visualized (Fig. 4). CT was also significant for bilateral pleural effusion and subcentimetric mediastinal lymph nodes. Based on histopathology and imaging, diagnosis of primary angiosarcoma of chest wall with pulmonary metastasis was reached. The patient was referred to surgery department for further management. As the patient presented at a very advanced stage (stage IV), surgery was not an option. He was started on chemotherapy in the oncology department but died within 3 months of starting treatment.

\section{DISCUSSION}

Angiosarcoma is a very rare mesenchymal tumor accounting for $1-2 \%$ of all soft-tissue sarcomas [4].

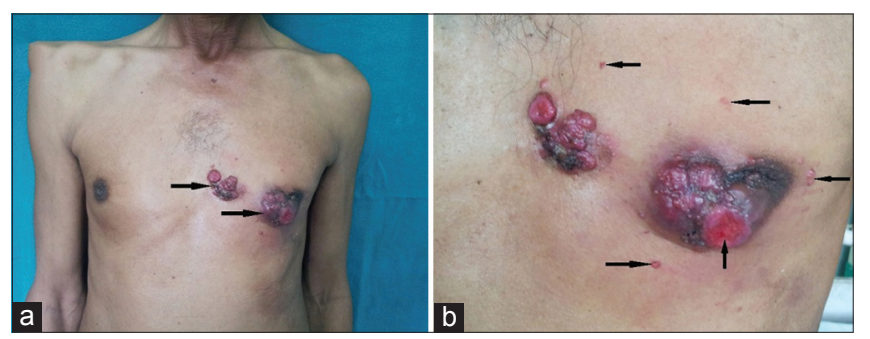

Figure 1: (a) Two fungating growths over left anterior chest wall (arrows); (b) Ulcer on the surface of larger plaque along with discrete papular lesions in the vicinity (arrows).

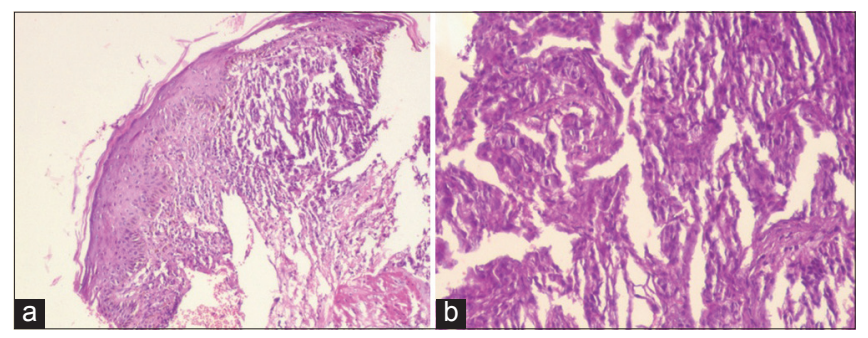

Figure 2: Photomicrograph showing: (a) Mild acanthosis of the epidermis, dermis contains vascular tumor with slit-like spaces (100 × H\&E); (b) Slit-like vascular channels lined by large pleomorphic cells having vesicular nuclei and prominent nucleoli $(400 \times \mathrm{H} \& \mathrm{E})$.

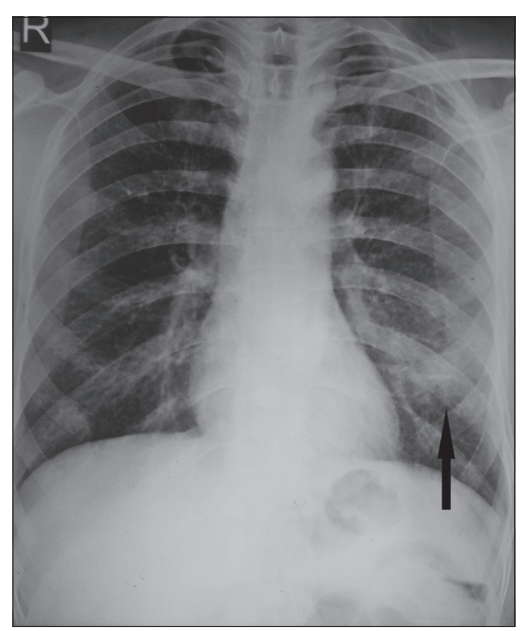

Figure 3: Chest X-ray showing area of haziness in left lower lung zone (arrow).

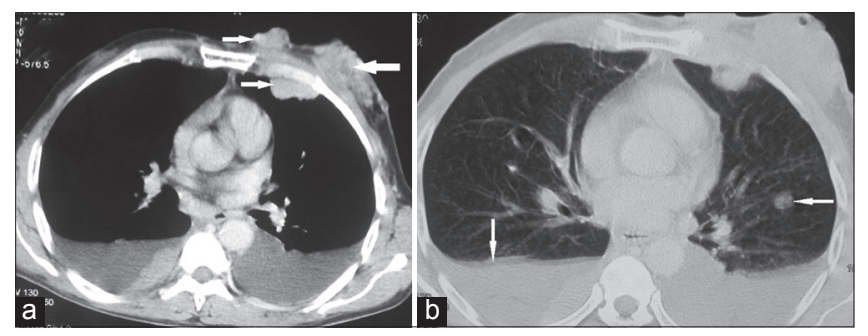

Figure 4: CECT chest showing: (a) A heterogeneous enhancing lesion with non- enhancing central area of necrosis in left chest wall with muscular invasion, infiltration into the overlying skin \& two small well defined enhancing nodules medial to the main lesion (arrows); (b) Bilateral pleural based soft tissue densities along with multiple secondaries in both lung fields \& bilateral pleural effusion (arrows). 
It can be primary i.e arising de novo or secondary to irradiation, trauma, lymphedema [5]. The common sites affected are skin, soft tissue, liver, spleen, heart and breast [6]. Primary angiosarcoma of chest wall can originate from bone, soft tissue or cartilage of chest wall and account for $5 \%$ of all thoracic neoplasms [7]. Chronic lymphedema, irradiation and exposure to vinyl chloride, thorostat, arsenic, anabolic steroid or foreign bodies are considered as main culprits [8]. None of these factors were present in our patient who was diagnosed as de novo case of primary angiosarcoma of chest wall. The clinical presentation of PACW can vary from chest pain, dyspnoea, hemothrax, chest wall mass or can be totally asymptomatic [9] (as in our case).

In our patient, the first impression was of primary cutaneous angiosarcoma (PCA) but as chest is not a common site for PCA, we considered other possibilities and further evaluated the patient. Histopathology was consistent with angiosarcoma. To rule out any metastasis to internal organs, chest $\mathrm{x}$-ray, CECT and USG abdomen and pelvis were requested. CECT revealed a heterogeneous enhancing mass in the left anterior chest wall with invasion into sub-cutaneous fat planes and infiltrating the overlying skin. Therefore, diagnosis of chest wall angiosarcoma was reached by co-relating clinical, radiological and pathological findings.

We did not come across any such presentation in dermatological literature. Such rare clinical presentations may often confront dermatologists and dermatosurgeons. This emphasizes their role in keeping a high index of suspicion in such cases and also the importance of referring the patient to other specialities relevant to the condition to expedite the diagnosis. This would lead to timely intervention and increment survival as these tumors have bad prognosis owing to their aggressive nature and associated mortality.

\section{Gold Standard and Advances in the Treatment of Angiosarcomas}

Angiosarcomas are very aggressive tumors, so treatment should be started early once diagnosis is established. As regards the therapy, it is important to underline that Angiosarcoma treatment has to be planned by a multidisciplinary team of medical experts drawn from all the involved disciplines [10].

\section{Surgical Intervention}

In operable patients with localised disease, surgical resection (en bloc) with the aim of obtaining negative surgical margins is the primary mode of treatment [11].

\section{Chemotherapy}

Cytotoxic chemotherapy forms the cornerstone of treatment for locally advanced inoperable or metastatic angiosarcoma. The therapeutic goals are to achieve control over the disease, to stop or postpone disease progression and to achieve or maintain symptom control for prolonged periods of time [12].

\section{Doxorubicin}

The first-line chemotherapy for advanced, metastatic or non-resectable soft tissue sarcoma is based on anthracyclines, and the most frequently used compound is doxorubicin [13]. The response rate to doxorubicin as a single agent or in combination is reported to range between $40 \%$ and $65 \%[14,15]$. The major adverse effect associated with doxorubicin is cardiomyopathy [12].

\section{Ifosfamide}

Ifosfamide, a cytotoxic alkalizing agent is used as a second line drug when doxorubicin has failed or is contraindicated. Ifosmide is usually given at a dose of $8-12 \mathrm{~g} / \mathrm{m}^{2}$ per cycle equally fractioned as single doses over 3-5 days. It achieves results comparable to doxorubicin but it is accocaited with number of severe adverse effects including leucopenia, neutopenia, renal toxicity and encephalopathy [12].

\section{Paclitaxel}

Paclitaxel has specific exquisite efficacy in angiosarcoma [12]. The activity of paclitaxel in angiosarcoma had been confirmed by a phase II trial assessing the efficacy of the weekly paclitaxel regimen (80 mg/m² dl, d8, dl 5, 21-day-cycle) [16].

\section{Gemcitabine}

Only a few anecdotal responses to gemcitabine monotherapy have been reported in angiosarcoma previously treated with anthracyclines and paclitaxel. Tolerability of gemcitabine plus docetaxel is fair, with less cardiac toxicity compared with anthracyclines $[17,18]$.

\section{Bevacizumab}

Bevacizumab, recombined humanized monoclonal antibody against vascular endothelial growth factor 
(VEGF), has emerged as a potential therapeutic option for angiosarcoma management [19-21]. In a phase 2 trial $(\mathrm{N}=26)$, bevacizumab produced a $12 \%$ $\mathrm{PR}$ rate in patients with angiosarcoma and epithelioid hemangioendothelioma [22].

\section{Pazopanib}

Pazopanib, a tyrosine kinase inhibitor is the first non-chemotherapeutic anticancer agent approved by regulatory authorities for soft tissue sarcoma. Pazopanib acts by interfering with the vascular endothelial growth factor and platelet-derived growth factor pathways. The approval of this oral anti-angiogenic agent is based on the EORTC trial 62072 (PALETTE) $[23,24]$.

\section{Other Chemotherapeutic Drugs}

Other non-approved treatment options for angiosarcomas include multikinase inhibitors (eg, sorafenib and sunitinib). In phase 2 trials, sorafenib produced a $14 \% \mathrm{PR}$ rate and a median OS of 14.3 months in an angiosarcoma trial $(\mathrm{n}=37),[25]$ whereas sunitinib did not produce a response rate in patients with angiosarcoma $(n=2)$ or fibrous tumor/ hemangiopericytoma $(n=3)$ [26].

\section{Radiation Therapy}

Preoperative radiation followed by resection is advised in borderline resectable cases. The dose recommendations include 45 to 50 Gy for undissected subclinical disease, 60 to 65 Gy for a postoperative tumor bed with positive microscopic margins, and 70 to 75 Gy for gross disease [27]. Adjuvant radiation with or without chemotherapy is indicated for patients with high-grade STS [stage II-III; American Joint Committee on Cancer, Cancer Staging Manual, Seventh Edition (2010)]. Alternatively, these modalities may be delivered preoperatively to reduce tumor size or improve resectability, particularly in potentially resectable cases or when there are concerns for adverse functional outcomes after surgery [28].

\section{International Therapeutics Guidelines}

A number of academic organisations have published guidelines for the treatment of inoperable, advanced, metastatic sarcoma.

\section{European society for medical oncology (ESMO)}

ESMO guidelines recommend as first-line treatment anthracyclines as single agent or in combination with ifosfamide or single-agent ifosfamide if there are specific contra-indications. Second-line treatments include ifosfamide at standard doses if patients have not been previously treated with this agent during first-line treatment. A high-dose ifosfamide schedule is recommended by ESMO if the drug had been previously used at a lower dose [29].

\section{British sarcoma group (BSG)}

BSG recommends single-agent doxorubicin or ifosfamide or doxorubicin and ifosfamide in the firstline setting. It recommends second-line treatments with either ifosfamide, trabectedin, gemcitabine and docetaxel or the older drug dacarbazine [13].

\section{National comprehensive cancer network (NCCN)} NCCN guidelines recommend paclitaxel and bevacizumab as treatment options for patients with angiosarcoma [28].

There have been some promising developments in the areas of immunotherapy, vaccine therapy, adoptive immunotherapy, immune synapse blockade and antibody therapy in soft tissue sarcomas but mostly remains experimental. Current clinical experience with these agents/regimen is too, limited to draw any conclusions [30].

The major drawback is the paucity of randomised trials with only a few retrospective case series or case reports, all suggesting that among soft tissue sarcomas, angiosarcoma appears to be more sensitive to cytotoxic chemotherapy. The rarity of angiosarcoma represents a major limitation to the randomized trials $[1,10]$.

Despite all therapeutic efforts, the patients prognosis is still unfavourable $[31,32]$. As our patient presented at a very advanced stage, surgery was not an option. He was started on chemotherapy with cytotoxic drugs but unfortunately he succumbed to the disease within three months of starting the treatment.

\section{CONCLUSION}

Primary angiosarcoma of chest wall remains a rare medical condition and its invasion to skin is an exceptionally rare phenomenon. Our case illustrates the insidious nature and cutaneous invasive potential of angiosarcoma with diagnosis only in late stages which harbours worst prognosis with death coming within few months. Medical practitioners in general and dermatologists in particular are likely to encounter 
such presentation of internal diseases manifesting in the skin which therefore serves as a window to systemic diseases.

\section{REFERENCES}

1. Young RJ, Brown NJ, Reed MW, Hughes D, Woll PJ. Angiosarcoma. Lancet Oncol. 2010;11:983-91.

2. Khodayari A, Khojasteh A. Mandibular pathologic fracture as a first sign of disseminated angiosarcoma: A case report and review of literatures. Oral Oncol Extra. 2005;41:178-82.

3. Bocklage T, Leslie K, Yousem S, Colby T. Clinical and Pathologic Features of Twenty-One Cases. Mod Pathol. 2001;14:1216-25.

4. Andersen NJ, Froman RE, Kitchell BE, Duesbery NS. Clinical and Molecular Biology of Angiosarcoma. In: Derbal F, ed. Soft Tissue Tumours. InTech. 2011;149-174.

5. Weiss SW, Goldblum JR. Malignant vascular tumors. In: Weiss SW, Goldblum JR, ed. Enzinger and Weiss's soft tissue tumors. St. Louis: Mosby; 2001; 917-54.

6. Patel AM, Ryu JH. Angiosarcoma in the lung. Chest. 1993;103:1531-5.

7. Hsu PK, Lee HC, Hsieh CC, Wu YC, Wang LS, Huang BS, et al. Management of primary chest wall tumors: 14 years' clinical experience. J Chin Med Assoc. 2006;69:377-82.

8. Kim DW, Lee KN, Lee SY, Roh MS. Angiosarcoma of the Chest Wall associated with Chronic Empyema and Pulmonary Metastasis: A Case Report. J Korean Soc Radiol. 2011;64:45-8.

9. Alexiou C, Clelland CA, Robinson D, Morgan WE. Primary angiosarcomas of the chest wall and pleura. Eur J Cardiothorac Surg. 1998;14:523-6.

10. Hillenbrand T, Menge F, Hohenberger P, Kasper B. Primary and secondary angiosarcomas: a comparative single-center analysis. Clin Sarcoma Res. 2015;5:14.

11. Zemanova M, Machalekova K, Sandorova M, Boljesikova E, Skultetyova M, Svec J, et al. Clinical management of secondary angiosarcoma after breast conservation therapy. Rep Pract Oncol Radiother. 2014;19:37-46.

12. Schöffski P, Cornillie J, Wozniak A, Li H, Hompes D. Soft tissue sarcoma: an update on systemic treatment options for patients with advanced disease. Oncol Res Treat. 2014;37:355-62.

13. Grimer R, Judson I, Peake D, Seddon B. Guidelines for the management of soft tissue sarcomas. Sarcoma. 2010;2010:506182.

14. Budd GT. Management of angiosarcoma. Cur Oncol Rep. 2002;4:515-9.

15. Fury MG, Antonescu CR, Van Zee KJ. A 14-year retrospective review of angiosarcoma: clinical characteristics, prognostic factors, and treatment outcomes with surgery and chemotherapy. Cancer J. 2005;11:241-7.

16. Penel N, Binh NB, Bay JO, Cupissol D, Ray-Coquard I, Piperno-Neumann S, et al. Phase II trial of weekly paclitaxel for unresectable angiosarcoma: the AngioTax study. J Clin Oncol. 2008;26:5269-74.

17. Hensley ML, Maki R, Venkatraman E. Gemcitabine and docetaxel in patients with unresectable leiomyosarcoma: results of a phase II trial. J Clin Oncol. 2002;20:2824-31.

18. Maki RG, Wathen JK, Patel SR. For SARC, the Sarcoma Alliance for Research through Collaboration Randomized phase II study of gemcitabine and docetaxel versus gemcitabine alone in patients with metastatic soft-tissue sarcomas: results of sarcoma alliance for research through collaboration study 002. J Clin Oncol. 2007;25:2755-63.

19. Koontz BF, Miles EF, Rubio MA, et al. Preoperative radiotherapy and bevacizumab for angiosarcoma of the head and neck: two case studies. Head Neck 2008;30:262-6.

20. Fuller CK, Charlson JA, Dankle SK, Russell TJ. Dramatic improvement of inoperable angiosarcoma with combination paclitaxel and bevacizumab chemotherapy. J Am Acad Dermatol. 2010;63:e83-4.

21. De Yao JT, Sun D, Powell AT, Rehmus EH. Scalp angiosarcoma remission with bevacizumab and radiotherapy without surgery: A case report and review of the literature. Sarcoma. 2011;2011:160369.

22. Agulnik M, Okuno SH, Von Mehren M, von Mehren M, Jovanovic BD, Brockstein BE, et al. An open-label multicenter phase II study of bevacizumab for the treatment of angiosarcoma. Ann Oncol. 2013;24:257-63.

23. Hurwitz HI, Dowlati A, Saini S, Savage S, Suttle AB, Gibson DM, et al. Phase I trial of pazopanib in patients with advanced cancer. Clin Cancer Res. 2009;15:4220-7.

24. Sleijfer S, Ray-Coquard I, Papai Z, Le Cesne A, Scurr M, Schöffski P, et al. Pazopanib, a multikinase angiogenesis inhibitor, in patients with relapsed or refractory advanced soft tissue sarcoma: A phase II study from the European Organisation for Research and Treatment of Cancer Soft Tissue and Bone Sarcoma Group (EORTC study 62043). J Clin Oncol. 2009;27:3126-32.

25. Maki RG, D’Adamo DR, Keohan ML, Saulle M, Schuetze SM, Undevia SD, et al. Phase II study of sorafenib in patients with metastatic or recurrent sarcomas. J Clin Oncol. 2009;27:3133-40.

26. George S, Merriam P, Maki RG, Van den Abbeele AD, Yap JT, Akhurst T, et al. Multicenter phase II trial of sunitinib in the treatment of nongastrointestinal stromal tumor sarcomas. J Clin Oncol. 2009;27:3154-60.

27. Scott MT, Portnow LH, Morris CG, Marcus Jr RB, Mendenhall NP, Mendenhall WM, Indelicato DJ. Radiation therapy for angiosarcoma: the 35-year University of Florida experience. Am J clin Oncol. 2013;36:174-80.

28. National Comprehensive Cancer Network. NCCN Clinical Practice Guidelines in Oncology. Soft Tissue Sarcoma, Vol. 1, 2011. 2011. Available at http:/ /www.nccn.org. Accessed July 26, 2016.

29. The ESMO/European Sarcoma Network Working Group. Soft tissue and visceral sarcomas: ESMO Clinical Practice Guidelines for diagnosis, treatment and follow-up. Ann Oncol. 2012;23(Suppl 7):viii92-9.

30. Linch M, Miah AB, Thway K, Judson IR, Benson C. Systemic treatment of soft-tissue sarcoma- gold standard and novel therapies. Nat Rev Clin Oncol. 2014;11:187-202.

31. Buehler D, Rice SR, Moody JS, Rush P, Hafez GR, Attia S, et al. Angiosarcoma outcomes and prognostic factors: a 25-year single institution experience. Am J Clin Oncol. 2014;37:473-9.

32. Lahat G, Dhuka AR, Hallevi H, Xiao L, Zou C, Smith KD et al. Angiosarcoma: clinical and molecular insights. Ann Surg. 2010;251:1098-106.

Copyright by Sabha Mushtaq, et al. This is an open-access article distributed under the terms of the Creative Commons Attribution License, which permits unrestricted use, distribution, and reproduction in any medium, provided the original author and source are credited.

Source of Support: Nil, Conflict of Interest: None declared. 